\title{
Expression of TNFRSF6B in kidneys is a novel predictor for progression of chronic kidney disease
}

\author{
Wei-Cheng Tseng ${ }^{1}$, Wu-Chang Yang ${ }^{2}$, An-Hang Yang ${ }^{3}$, Shie-Liang Hsieh ${ }^{4,5,6,7}$ and \\ Der-Cherng Tarng $2,4,5,7,8$
}

${ }^{1}$ Division of Nephrology, Department of Medicine, Taipei City Hospital Heping Fuyou Branch, Taipei, Taiwan; ${ }^{2}$ Division of Nephrology, Department of Medicine, Taipei Veterans General Hospital, Taipei, Taiwan; ${ }^{3}$ Department of Pathology, Taipei Veterans General Hospital, Taipei, Taiwan; ${ }^{4}$ Immunology Center, Taipei Veterans General Hospital, Taipei, Taiwan; ${ }^{5}$ Institute of Clinical Medicine, National Yang-Ming University, Taipei, Taiwan; ${ }^{6}$ Institute of Microbiology and Immunology, National Yang-Ming University, Taipei, Taiwan; ${ }^{7}$ Infection and Immunology Research Center, National Yang-Ming University, Taipei, Taiwan and

${ }^{8}$ Department and Institute of Physiology, National Yang-Ming University, Taipei, Taiwan

\begin{abstract}
TNFRSF6B overexpression in tumors is a novel predictor for poor prognosis in various cancers; however, whether TNFRSF6B could be expressed in kidney tissues of patients with chronic kidney disease is unknown. Current established risk factors cannot fully predict the progression of chronic kidney disease, and, therefore, it is mandatory to develop a newer marker for predicting disease progression. We conducted a prospective cohort study comprised 167 patients with chronic kidney disease undergoing renal biopsy at a tertiary hospital with median follow-up of $\mathbf{3 0 . 5}$ months. Computer-assisted quantitative immunohistochemical staining analysis of TNFRSF6B in kidney tissues, the expression of $\alpha$-smooth muscle actin and percentage of fibrosis in renal interstitium, estimated glomerular filtration rate, and urinary protein excretion rate were investigated. Study endpoint was a doubling of serum creatinine and/or end-stage renal failure requiring renal replacement therapy. We found that TNFRSF6B was predominantly expressed in the tubular epithelial cells of renal cortex. The higher the expression of TNFRSF6B, the more the expression of $\alpha$-smooth muscle actin and fibrosis in interstitium $(P<0.001)$. Forty patients reaching endpoint had lower baseline estimated glomerular filtration rate and higher expression of TNFRSF6B in renal tubular epithelial cells. Multivariate Cox regression analysis showed that high expression of TNFRSF6B independently predicted the risk toward the renal endpoint with a hazard ratio of 3.46 (95\% confidence interval $(\mathrm{Cl}) 1.76-6.80, P<0.001)$ by adjusting for clinical and pathologic variables. While added to a model of estimated glomerular filtration rate, proteinuria and other conventional risk factors, TNFRSF6B further significantly improved the model predictability for progression of chronic kidney disease (area under the curve, 0.82 ). In conclusion, TNFRSF6B is associated with renal fibrosis and high expression of TNFRSF6B is a novel biomarker for predicting the progression of chronic kidney disease.
\end{abstract}

Modern Pathology (2013) 26, 984-994; doi:10.1038/modpathol.2013.29; published online 1 March 2013

Keywords: chronic kidney disease; kidney disease progression; renal fibrogenesis; TNFRSF6B

Chronic kidney disease is an emerging worldwide public health problem. The prevalence (10-12\%) of chronic kidney disease is high in the United States,

Correspondence: Professor D-C Tarng, MD, PhD, Institutes of Physiology and Clinical Medicine, National Yang-Ming University, Taipei, and Division of Nephrology, Department of Medicine and Immunology Center, Taipei Veterans General Hospital, 201, Section 2, Shih-Pai Road, Taipei 11217, Taiwan. E-mail: dctarng@vghtpe.gov.tw

Received 5 September 2012; revised 21 November 2012; accepted 22 November 2012; published online 1 March 2013
Europe, and Asia-Pacific region. ${ }^{1}$ In the early chronic kidney disease, a substantial portion of the patients suffered from anemia, cardiovascular disease, premature death, and even progression to end-stage renal failure. ${ }^{2-4}$ Therefore, it is of most importance to discover new markers for identification of patients at high risk of progression of chronic kidney disease. Although hypertension, hyperglycemia, hyperlipidemia and proteinuria are well-known established risk factors for predicting renal function decline, these elements do not fully explain the disease progression. ${ }^{5-7}$ Therefore, it 
highlights our need for the new biomarkers to identify patients with chronic kidney disease at higher risk for progression.

TNFRSF6B (also known as decoy receptor 3) belongs to the tumor necrosis factor receptor family, but lacks transmembrane and cytoplasmic domains in its sequence. ${ }^{8}$ TNFRSF6B could act as decoy receptor to neutralize the proapoptotic effects of Fas ligand, LIGHT, and tumor necrosis factorlike molecule $1 \mathrm{~A}$ by blocking the interaction with their respective receptors and evading immune surveillance. ${ }^{8-10}$ Overexpression of TNFRSF6B, originally found in lung and colon cancers and virus-associated lymphoma, is capable of protecting cancer cells against apoptosis. ${ }^{11}$ Mounting evidences have shown that TNFRSF6B overexpression is a novel tissue biomarker for predicting tumor invasion and worsening of various chronic inflammatory diseases. ${ }^{11}$ Serum TNFRSF6B level is markedly elevated in patients with chronic kidney disease, ${ }^{12}$ and our recent study has shown that serum TNFRSF6B is an inflammatory biomarker and associates with mortality in hemodialysis patients. ${ }^{13}$ In human tissues, TNFRSF6B is normally expressed in the colon, stomach, spleen, lymph nodes, and lung but not in kidneys. ${ }^{8,14}$ Till now, the expression of TNFRSF6B in kidney tissues of patients with chronic kidney disease and its pathophysiologic role in human kidney disease have never been well studied.

Tubulointerstitial fibrosis is the ultimate common pathway of chronic kidney disease irrespective of the original etiologies. ${ }^{15}$ Interstitial fibroblasts are currently considered the principal effector cells responsible for renal fibrosis. ${ }^{16}$ Fibroblasts in response to initial insults should be eliminated by apoptosis in later stages of wound healing physiologically. Otherwise, excessive deposition of extracellular matrix supervenes and leads to eventual renal fibrosis. ${ }^{17}$ Anti-apoptotic effect of TNFRSF6B has been shown to decrease elimination of rheumatoid synovial fibroblasts by blocking the interaction with Fas ligand. ${ }^{18}$ Fas is normally expressed in human renal interstitial fibroblasts ${ }^{19}$ and we hypothesize that TNFRSF6B could involve in the renal fibrogenesis. Therefore, it prompts us to investigate whether TNFRSF6B is pathologically expressed in the kidney tissues of patients with chronic kidney disease, and to appraise whether expression of TNFRSF6B in kidneys is a newer tissue biomarker for prediction of disease progression.

\section{Materials and methods}

\section{Study Population}

From January 2005 to March 2008, we enrolled 167 consecutive adult patients undergoing percutaneous needle kidney biopsy for nephrotic syndrome, nephritis, or unexplained renal failure at Taipei
Veterans General Hospital. Recruited patients had age of $>20$ years and had one or more reasons for renal biopsy, such as cellular casts in urine for $>3$ months, daily protein excretion rate of $>1 \mathrm{gm}$ for $>3$ months, or kidney injury of unknown origin and not due to prerenal azotemia, nephrotoxic agents, septicemia, or local kidney infections, or urinary tract obstruction. Exclusion criteria were dialysis patients or renal transplant recipients, active malignancy, rare kidney diseases including Alport's syndrome, lipoprotein glomerulopathy, thin basement membrane disease and immunotactoid glomerulopathy, insufficient specimen amount of biopsy, and lacked informed consents. Sixty patients were lost to follow-up and withdrew from the study because they moved away or were not referred by their physicians for follow-up visits in the kidney unit. Finally, a total of 107 patients from baseline cohort could be assessed the follow-up till May 2009. Control groups comprised six subjects receiving nephrectomy for localized circumscribed kidney tumor, with normal renal function and no other significant comorbidities. Normal renal tissue was obtained from the uninvolved pole of the removed kidneys. The study was approved by the Committee on Human Research of Taipei Veterans General Hospital. Written informed consent was obtained from each of the enrolled study patients and control subjects.

Baseline renal function tests, urine protein excretion rate, and demographic data including age, sex, smoking status, comorbidities, and medications were recorded at the time of biopsy. Renal function tests were regularly checked in follow-up visits in the kidney unit. All patients were enrolled by one physician to minimize interobserver variations. This was complemented by clinical assessment of body weight, body mass index, blood pressure, and fasting blood glucose. Diabetes was diagnosed on the basis of the World Health Organization criteria. Hypertension was defined as blood pressure $>140 / 90 \mathrm{~mm} \mathrm{Hg}$ and/or the use of antihypertensive medication. Antihypertensive drugs were taken by $78(73 \%)$ patients: angiotensin-converting enzyme inhibitors and/or angiotensin-II receptor blockers $(n=69)$, calcium channel blockers $(n=49), \quad \beta$-receptor blockers $\quad(n=30), \quad$ and $\alpha-1$ receptor blockers $(n=11)$.

\section{Histopathology}

Formalin-fixed, paraffin-embedded kidney biopsy specimens of 107 patients and normal kidney tissues of six control subjects were sectioned in $4 \mu \mathrm{m}$ and stained with hematoxylin and eosin for histological examination. To determine the percentage of interstitial fibrosis, sections were stained with a Masson's trichrome kit (Accustain, SigmaAldrich, St Louis, MO, USA) according to the manufacturer's instructions. The percentage of 
global obsolescence of glomeruli and the severity of tubulointerstitial injury were examined under 20 randomly selected high-power fields $(\times 400)$. Glomerulosclerosis was determined using a percentage of glomeruli expressing global or segmental sclerosis. Tubular damage, fibrosis and inflammatory cell infiltration in cortical interstitium were graded by a scoring system from 0 to 4 (0, no changes; 1 , changes affecting $<25 \% ; 2$, changes affecting $25-50 \%$; 3 , changes affecting $50-75 \%$; and 4 , changes affecting $>75 \%$ of the cortical parenchyma) as previously described. ${ }^{20,21}$ All sections were analyzed by a pathologist (AHY) unaware of the clinical and laboratory data.

\section{Immunohistochemistry}

Consecutive parallel sections from all patients and control subjects were stained with mouse monoclonal antibodies directly against human TNFRSF6B (diluted 1:20; BioLegend, San Diego, CA, USA), and $\alpha$-smooth muscle actin (diluted 1:500; DakoCytomation, Glostrup, Denmark). Immunohistochemical staining was performed as previously described. ${ }^{21}$ Briefly, after deparaffinization and rehydration, $4-\mu \mathrm{m}$ sections were subjected to heat antigen retrieval in a microwave oven $(650 \mathrm{~W}$, $12 \mathrm{~min}$ ) in $10 \mathrm{mM}$ sodium citrate ( $\mathrm{pH}$ 6.0), after which endogenous peroxidase activity was blocked by $3 \%$ hydrogen peroxide. Thereafter, tissue sections were incubated with the primary antibodies at $4{ }^{\circ} \mathrm{C}$ overnight and then with secondary antibody (Envision + Dual Link System-HRP, DakoCytomation) for $30 \mathrm{~min}$ at room temperature. Signals were developed with 3-amino-9-ethyl carbazole (DakoCytomation) for $10 \mathrm{~min}$ and counterstained with hematoxylin. The primary antibody was replaced by isotype-matched antibody with constant all other experimental conditions as a negative control. Staining of each study sample was compared with the staining of control slides (the same control subject) on that run to circumvent run-to-run variation of staining intensity.

Cytoplasmic staining for TNFRSF6B and peritubular interstitial staining for $\alpha$-smooth muscle actin were defined as positive staining. Computerassisted quantitative analysis (Image Pro Plus 6.0, MediaCybernetics, SilverSpring, MD, USA) was performed as previously described. ${ }^{22}$ Briefly, 5 glomeruli and 20 randomly selected nonoverlapping high-power fields of each renal cortical section were captured by a PROVIS AX80 microscope (Olympus, Tokyo, Japan) equipped with a 12-bit RETIGA 1300 digital camera (QImaging, Burnaby, Canada). The captured images were then analyzed by Image Pro Plus software. To eliminate variation, lighting intensity and optical setting of the microscope during image capturing were kept constant on any given day. The intensity at the blank area of slides and the intensity while the light was off were used as maximal incident light level and black level for calibration of optical density, respectively. Two color segmentations, one identified immunopositive red cells and the other recognized blue background cells, were then applied. Integrated optical density was calculated as the intensity of red pixels multiplied by their pixel area. Glomerulus and tubulointerstitium were analyzed separately. Quantitative immunohistochemical staining value was calculated as the integrated optical density divided by total area occupied by the red and blue cells in each slide. Arterioles, which indigenously expressed $\alpha$-smooth muscle actin, were not included in the quantification (Supplementary Figure S2).

\section{Laboratory Measurements}

Laboratory measurements were made on 12-h fasting venous blood samples before kidney biopsy. Creatinine, albumin, cholesterol and triglyceride in serum were measured with a Hitachi autoanalyzer 736-60 (Hitachi Ltd, Tokyo, Japan). Serum high-sensitivity C-reactive protein was measured by an immunoturbidimetric assay using a rate nephelometry (Beckman, Galway, Ireland). Urine protein excretion rate was determined on 24-h urine samples using an Olympus AU640 autoanalyzer (Olympus). Estimated glomerular filtration rate was calculated by the simplified modified diet for renal disease equation. ${ }^{23}$

\section{Prospective Follow-up}

Following the baseline investigations, patients were followed prospectively until the primary endpoint or the end of the observation period was reached. The primary endpoint was defined as doubling of baseline serum creatinine and/or end-stage renal failure requiring renal replacement therapy.

\section{Statistical Analysis}

Descriptive statistics included mean values \pm s.d. for continuous data and percentages for categorical data. The values for serum creatinine, estimated glomerular filtration rate, urine protein excretion rate, high-sensitivity C-reactive protein, and percentage of global obsolescence of glomeruli were not normally distributed and were expressed as medians with the interquartile ranges. For between-twogroup comparisons, the Student's $t$-test was used for normally distributed data and the Mann-Whitney rank sum test for data with a non-normal distribution. Categorical variables were compared using Pearson $\chi^{2}$-test. Receiver operating characteristics analysis was used to find the optimal cutoff value of TNFRSF6B quantitative immunohistochemical staining value for identifying the progression to renal endpoint, and to calculate the area under the curve for the predictors. To assess discrimination 
Table 1 Baseline demographic and laboratory data and histopathology in chronic kidney disease patients with or without progression during the follow-up period

\begin{tabular}{|c|c|c|c|}
\hline Parameter $^{\mathrm{a}}$ & $\begin{array}{l}\text { Nonprogressors } \\
\quad(\mathrm{n}=67)\end{array}$ & $\begin{array}{l}\text { Progressors } \\
\quad(\mathrm{n}=40)\end{array}$ & $\mathrm{P}$-value \\
\hline \multicolumn{4}{|l|}{ Demographics } \\
\hline Age (years) & $50.0 \pm 19.1$ & $48.7 \pm 17.9$ & 0.71 \\
\hline Male gender $(n(\%))$ & $40(60)$ & $19(48)$ & 0.22 \\
\hline Current smoker (n (\%)) & $12(18)$ & $6(15)$ & 0.70 \\
\hline Diabetes $(n(\%))$ & $15(22)$ & $14(35)$ & 0.16 \\
\hline Hypertension ( $(\%)$ ) & $35(52)$ & $21(53)$ & 0.98 \\
\hline Etiology of renal disease & & & 0.25 \\
\hline \multicolumn{4}{|l|}{ Primary glomerulopathy } \\
\hline Minimal change disease $(n(\%))$ & $7(10)$ & $3(7.5)$ & \\
\hline Focal segmental glomerulosclerosis $(n(\%))$ & $11(16)$ & $5(12.5)$ & \\
\hline Membranous glomerulopathy (n (\%)) & $12(18)$ & $4(10)$ & \\
\hline Immunoglobulin A nephropathy (n (\%)) & $6(9)$ & $4(10)$ & \\
\hline Membranoproliferative glomerulonephritis (n (\%)) & $2(3)$ & $1(2.5)$ & \\
\hline \multicolumn{4}{|l|}{ Secondary glomerulopathy } \\
\hline Diabetic nephropathy (n (\%)) & $8(12)$ & $6(15)$ & \\
\hline Hypertensive nephrosclerosis (n (\%)) & $5(7)$ & $5(12.5)$ & \\
\hline Lupus nephritis (n (\%)) & $2(3)$ & $4(10)$ & \\
\hline Interstitial nephritis $(n(\%))$ & $14(21)$ & $8(19)$ & \\
\hline \multicolumn{4}{|l|}{ Blood pressure } \\
\hline Systolic (mm Hg) & $140 \pm 19$ & $142 \pm 18$ & 0.68 \\
\hline Diastolic (mm Hg) & $84 \pm 11$ & $84 \pm 8$ & 0.69 \\
\hline Body weight (kg) & $71.3 \pm 12.4$ & $68.1 \pm 9.5$ & 0.16 \\
\hline Body mass index $\left(\mathrm{kg} / \mathrm{m}^{2}\right)$ & $26.8 \pm 3.7$ & $26.0 \pm 3.8$ & 0.33 \\
\hline \multicolumn{4}{|l|}{ Laboratory data } \\
\hline Serum creatinine (mg/dl; median (interquartile range)) & $1.60(1.12-2.34)$ & $3.65(2.33-5.68)$ & $<0.001$ \\
\hline $\begin{array}{l}\text { Estimated glomerular filtration rate }\left(\mathrm{ml} / \mathrm{min} / 1.73 \mathrm{~m}^{2} \text {; median }\right. \\
{\text { (interquartile range) })^{\mathrm{C}}}\end{array}$ & $43.4(30.4-71.6)$ & $17.9(9.0-31.8)$ & $<0.001$ \\
\hline Urine protein (g/day/1.73 m²; median (interquartile range)) & $3.15(1.51-6.22)$ & $3.83(1.62-10.18)$ & 0.33 \\
\hline Serum albumin $(\mathrm{g} / \mathrm{dl})$ & $3.85 \pm 0.81$ & $3.31 \pm 0.75$ & 0.002 \\
\hline Serum cholesterol (mg/dl) & $260 \pm 91$ & $239 \pm 95$ & 0.26 \\
\hline Serum triglyceride (mg/dl) & $209 \pm 111$ & $219 \pm 195$ & 0.76 \\
\hline Serum high-sensitivity C-reactive protein (mg/dl; median (interquartile range)) & $3.60(1.09-4.32)$ & $4.31(1.02-5.21)$ & 0.71 \\
\hline \multicolumn{4}{|l|}{ Histopathology } \\
\hline Global obsolescence of glomeruli (\%) & $19.2 \pm 23.0$ & $45.3 \pm 36.7$ & $<0.001$ \\
\hline Interstitial fibrosis score & & & $<0.001$ \\
\hline $0(n(\%))$ & $18(27)$ & $6(15)$ & \\
\hline $1(n(\%))$ & $35(52)$ & $8(20)$ & \\
\hline $2(n(\%))$ & $6(9)$ & $7(18)$ & \\
\hline $3(n(\%))$ & $8(12)$ & $19(48)$ & \\
\hline Interstitial inflammation score & & & 0.004 \\
\hline $0(n(\%))$ & $21(31)$ & $14(35)$ & \\
\hline $1(n(\%))$ & $38(57)$ & $13(33)$ & \\
\hline $2(n(\%))$ & $5(7)$ & $2(5)$ & \\
\hline $3(n(\%))$ & $3(4)$ & $11(28)$ & \\
\hline \multicolumn{4}{|l|}{ Medications } \\
\hline $\begin{array}{l}\text { Angiotensin-converting enzyme inhibitor/ angiotensin-II receptor } \\
\text { blocker }(n(\%))\end{array}$ & $44(66)$ & $25(60)$ & 0.74 \\
\hline Immunosuppressant therapy $(n(\%))$ & & & 0.17 \\
\hline Corticosteroid ( $n(\%))$ & $46(68)$ & $19(47)$ & \\
\hline Cyclophosphamide (n (\%)) & $14(21)$ & $7(18)$ & \\
\hline Azathioprine $(n(\%))$ & $20(30)$ & $8(20)$ & \\
\hline Cyclosporine (n (\%)) & $15(22)$ & $11(28)$ & \\
\hline Mycophenolate (n (\%)) & $15(22)$ & $10(25)$ & \\
\hline
\end{tabular}

${ }^{a}$ Data were presented as mean \pm s.d. for normally distributed variables and as median and interquartile range for skewed variables where appropriate.

${ }^{\mathrm{b}}$ Comparison between two groups by Student's $t$-test or Mann-Whitney rank sum test for continuous variables as appropriate and Pearson $\chi^{2}$-test for categorical variables.

${ }^{\mathrm{C}}$ Estimated glomerular filtration rate was calculated by the simplified modified diet for renal disease formula. ${ }^{23}$

ability, $c$ statistic for model of conventional risk factors, including age, sex, smoking status, diabetes, body mass index, systolic blood pressure, and total cholesterol (base model), then with stepwise addition of urine protein, estimated glomerular filtration rate and TNFRSF6B quantitative immunohistochemical staining value (newer biomarker model) was calculated for prediction of progression 
of chronic kidney disease at 48 months of the study. ${ }^{24}$ Renal survival curve was generated by the Kaplan-Meier method. Differences in the time-toevent curves between patients with high and low expressions of TNFRSF6B in the renal tubular epithelial cells were compared by the log-rank test. Multivariate Cox proportional hazards model was used to assess the association between TNFRSF6B expression and progression of chronic kidney disease, adjusting for potential confounding factors. Any variables having a hazard ratio adjusted for age and gender with a $P<0.10$ (model 1) and the established risk factors for a poor renal outcome were considered as potential confounders and were adjusted for in the Cox regression analysis (model 2). A backward elimination procedure was performed using $P>0.05$ to remove any identified independent predictors for progression of chronic kidney disease. Statistical analysis was performed using the computer software Statistical Package of
Social Science (SPSS 12.0, 2003; SPSS Inc., Chicago, IL, USA). A $P$-value of $<0.05$ was considered statistically significant.

\section{Results}

\section{Baseline Characteristics of Patients}

Initially, we enrolled 167 patients with chronic kidney disease in the prospective cohort study. Sixty patients withdrew from the study because they were lost to follow-up. They did not differ significantly with respect to age, gender, and renal function at baseline as compared with those who completed the study.

The median follow-up after completion of the baseline investigation was 30.5 (interquartile range, 9.8 to 42.3 ) months. During the follow-up, there were 40 patients with progression to a renal end-
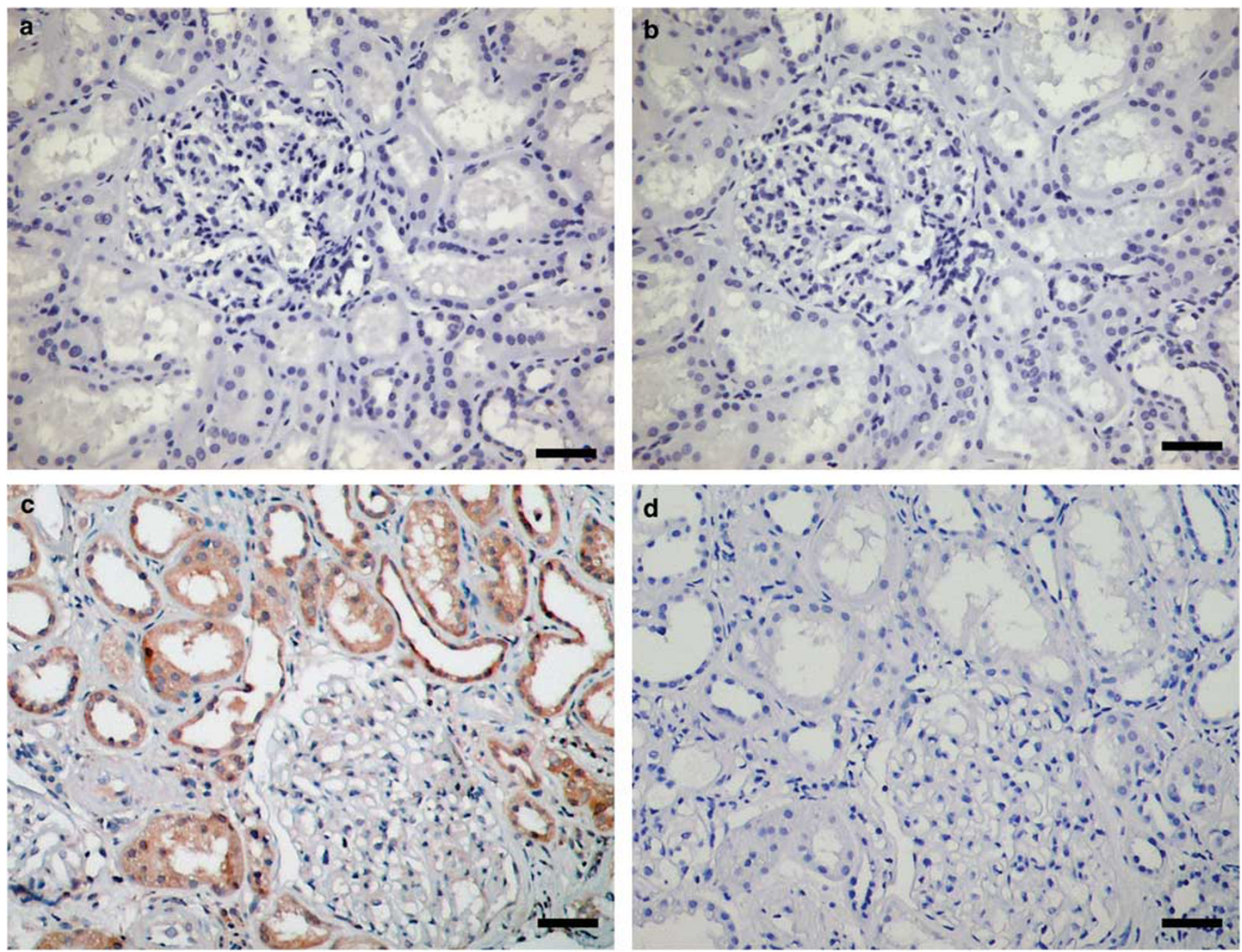

Figure 1 Immunohistochemical staining of TNFRSF6B expression in paraffin-embedded kidney biopsy sections. Representative images show that TNFRSF6B is not expressed in kidney tissue of normal controls (a). In chronic kidney disease (CKD) patients, TNFRSF6B is highly expressed (shown in red) in the tubular epithelial cells of renal cortex, but not in the vessels (asterisk), glomerulus or interstitium (c, e). Negative controls show no immunostaining of TNFRSF6B (b, d and f). Scale bar, $50 \mu \mathrm{m}$. The quantitative immunohistochemical staining value (QISV) of TNFRSF6B in renal tubules and glomeruli were assessed by computer-assisted quantitative staining analysis (g). Data are expressed as means \pm s.d. ${ }^{*} P<0.001$ versus glomeruli in CKD patients. 

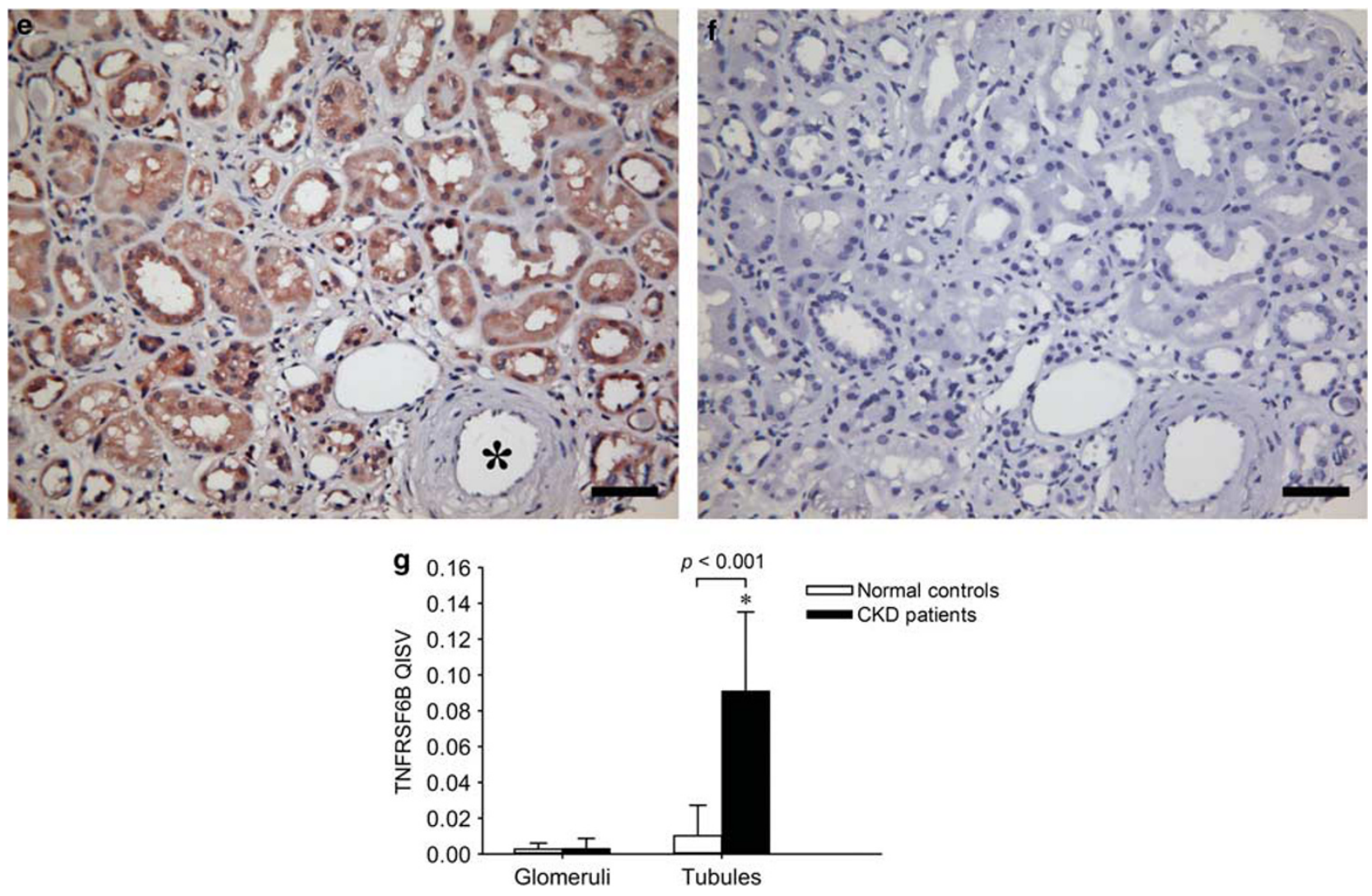

Figure 1 Continued.

point and 67 patients without progression. Among the progressors, 25 patients had doubled their serum creatinine and 15 patients had reached end-stage renal failure requiring renal replacement therapy. Progressors and nonprogressors were similar with respect to age, gender distribution, percentage of smoking status, diabetes, hypertension, etiology of renal disease, immunosuppressive treatment, and urine protein excretion. Nonetheless, the progressors had significantly higher serum creatinine and the percentage of glomerular obsolescence, interstitial fibrosis and inflammation scores, as well as lower estimated glomerular filtration rate and serum albumin at baseline (Table 1).

\section{TNFRSF6B Predominantly Expressed in Renal Tubular Epithelial Cells}

In kidney tissues of control subjects, TNFRSF6B immunoreactivity was undetectable (Figure 1a). Among patients with chronic kidney disease, TNFRSF6B staining was predominantly in the tubular epithelial cells of renal cortex but not in the glomeruli, interstitium, vessels, or tubular cells of renal medulla (Figures 1c and e and Supplementary Figure S3). In patients with chronic kidney disease, computer-assisted quantitative immunohistochemical staining value of TNFRSF6B in the renal tubular epithelial cells was significantly higher than that in the glomeruli. In control subjects, however, quantitative immunohistochemical staining value of TNFRSF6B was relatively low as compared with chronic kidney disease patients, and showed no difference in both renal tubular epithelial cells and glomeruli (Figure 1g).

\section{Expression of TNFRSF6B Associated with the Tissue Markers of Renal Fibrosis}

Optimal cutoff value of TNFRSF6B quantitative immunohistochemical staining value for predicting renal progression based on the receiver operating characteristics analysis was 0.1119 with the area under the curve of 0.64 (95\% CI 0.54 to 0.73). The study patients were then stratified into two groups with high $(n=31)$ and low $(n=76)$ expression of TNFRSF6B in renal tubular epithelial cells (Figure 2b). High TNFRSF6B expression group had significantly higher quantitative immunohistochemical staining value for $\alpha$-smooth muscle actin (Figure 2c) and percentage of interstitial fibrosis (Figure 2d) than low TNFRSF6B expression group among patients with chronic kidney disease. However, between the two groups of chronic kidney disease patients, no significant differences were observed in demographic and laboratory data such as serum creatinine, albumin, cholesterol, triglyceride, high-sensitivity C-reactive protein, and 
urine protein excretion rate at baseline, interstitial inflammation scores, as well as the renal etiology, in terms of primary glomerulonephritis, tubulointerstitial nephritis, and systemic diseases with kidney involvement.

\section{High TNFRSF6B Expression Independently Predicted Progression of Chronic Kidney Disease}

Forty progressors had significantly higher quantitative immunohistochemical staining value of TNFRSF6B in the renal tubular epithelial cells than 67 nonprogressors $(P=0.012$; Figure 3a). In the Kaplan-Meier curve of progression-free survival (Figure 3b), patients with high TNFRSF6B expression had worse renal survival $(P<0.001)$ and significantly shorter progression time compared with those with low expression: median time to progression 8.4 (interquartile range, 2.2 to 23.9) months compared with 16.5 (interquartile range, 6.1 to 33.4 ) months $(P<0.001)$, respectively.

In age- and gender-adjusted Cox regression analysis (model 1, Table 2), high expressions of TNFRSF6B and $\alpha$-smooth muscle actin, $>25 \%$ of fibrosis and $>50 \%$ of infiltrated inflammatory cells in interstitium, $>50 \%$ of glomerular obsolescence, lower baseline estimated glomerular filtration rate and use of immunosuppressant therapy were strongly associated with progression to renal endpoint (all $P<0.05$ ). In multivariate regression analysis (model 2, Table 2), high TNFRSF6B expression in renal tubular epithelial cells independently had a higher risk toward disease progression with a hazard ratio of $3.46(95 \%$ CI, $1.76-6.80, P<0.001)$ by adjusting for age, gender, and eleven other variables.

\section{Quantitative Immunohistochemical Staining Value of TNFRSF6B Significantly Increases the Predictability for Chronic Kidney Disease Progression}

To test discrimination ability, urine protein, estimated glomerular filtration rate and quantitative immunohistochemical staining value of TNFRSF6B were incorporated into the conventional risk factors (base model) for predicting chronic kidney disease progression (Table 3). The $c$ statistic of base model was 0.63. Adding urine protein to the base model only slightly increased $C$ statistic to 0.64 . With estimated glomerular filtration rate, urine protein, and conventional risk factors together in the model significantly increases the $c$ statistic to 0.76. Addition of quantitative immunohistochemical staining value of TNFRSF6B to urine protein, estimated a

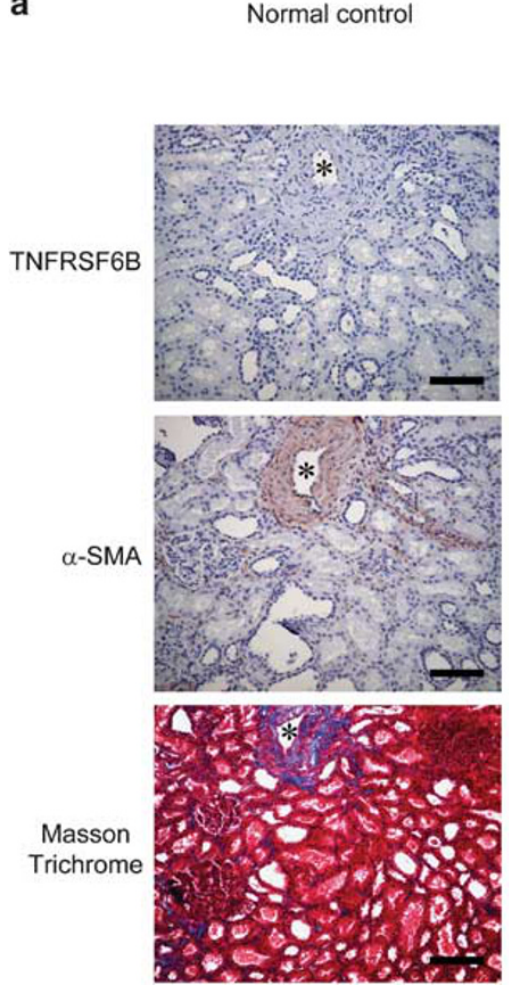

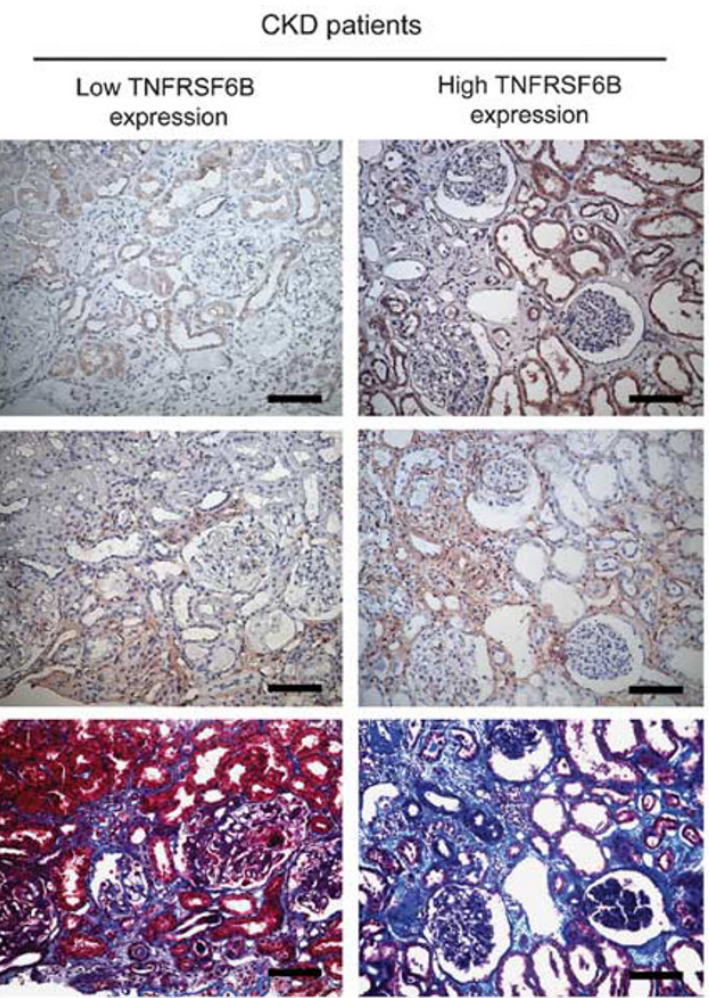
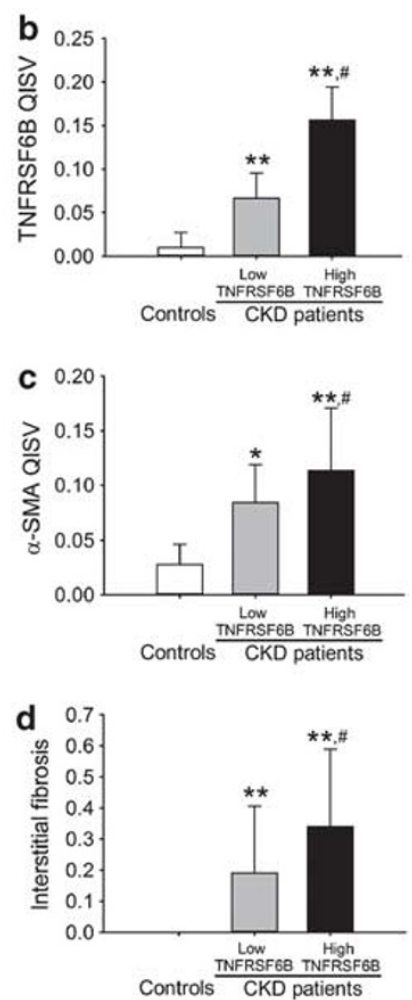

Figure 2 Representative photographs of immunohistochemical staining of TNFRSF6B, alpha-smooth muscle actin $(\alpha$-SMA) and Masson's trichrome staining in kidney tissues of chronic kidney disease (CKD) patients and normal controls (a). Asterisks denoted vessels. Scale bar, $100 \mu \mathrm{m}$. Quantitative immunohistochemical staining value (QISV) of TNFRSF6B (b), $\alpha$-smooth muscle actin (c) and the percentage of interstitial fibrosis (d) assessed by computer-assisted quantitative analysis. Patients were stratified into the high and low expression groups by the cutoff value 0.1119 of TNFRSF6B QISV based on the receiver operating characteristics curve analysis. Data were expressed as means \pm s.d. ${ }^{*} P<0.01{ }^{*} P<0.001$ versus normal controls; ${ }^{*} P<0.001$ vs low TNFRSF6B expression group. 


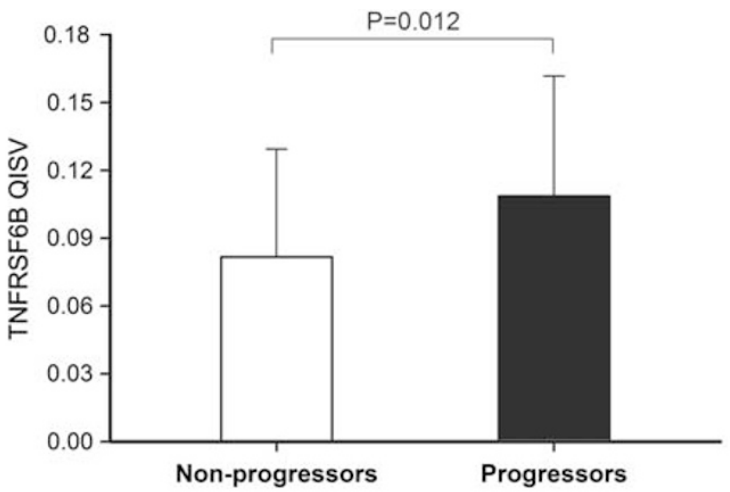

b

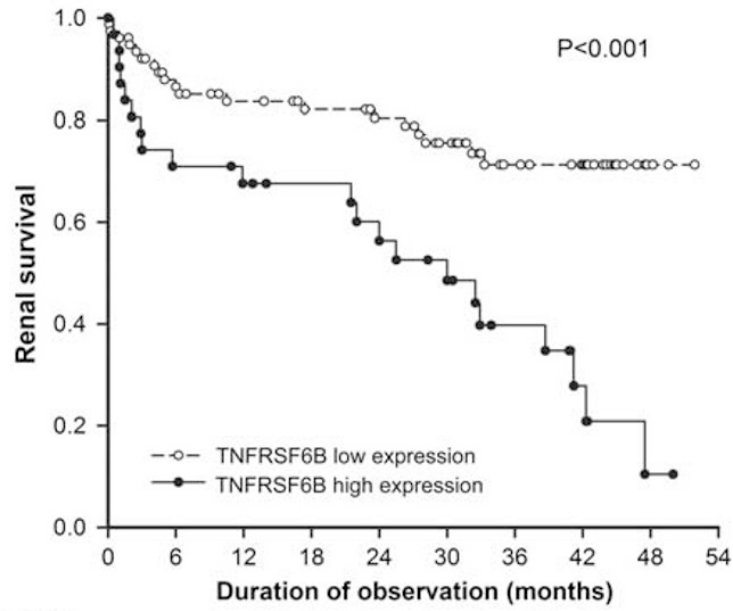

No. at risk

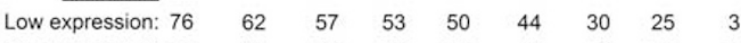

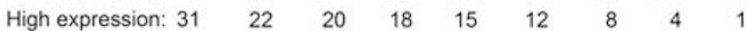

Figure 3 Difference of TNFRSF6B quantitative immunohistochemical staining value (QISV) between progressors and nonprogressors (a), and Kaplan-Meier curve for renal endpoint in patients with high and low expressions of TNFRSF6B in renal tubular epithelial cells (b). Patients were stratified into the high and low expression groups by the cutoff value of 0.1119 for TNFRSF6B QISV based on the receiver operating characteristics curve analysis.

glomerular filtration rate and conventional risk factors (newer marker model) further improve the model predictability with $C$ statistic of 0.82 $(P=0.001)$.

\section{Discussion}

This study, for the first time, showed that TNFRSF6B was pathologically expressed in the kidney tissue of patients with chronic kidney disease and not in that of subjects with normal kidney function. Elevated serum level of TNFRSF6B has been reported in patients with chronic kidney disease with indeterminate etiology. ${ }^{12}$ Interestingly, we also observed that TNFRSF6B expression could be evoked by tumor necrosis factor- $\alpha$ in a dose-dependent manner in HK-2 cells, a human proximal tubular cell line, and attenuated by SP600125, PD9805, and PDTC, suggesting that the proinflammatory cytokine, tumor necrosis factor- $\alpha$, participated in TNFRSF6B expression in renal proximal tubular epithelial cells (Supplementary Figure S1). Our data, therefore, do not support the notion that TNFRSF6B is filtered from the glomeruli and then engulfed into the renal tubular epithelial cells. In contrast, it is mainly due to the increased expression of TNFRSF6B in local renal tubular epithelial cells in response to renal inflammation characterized by the presence of proinflammatory cytokines in patients with chronic kidney disease.

In the present study, the most compelling finding is that high TNFRSF6B expression of renal tubular epithelial cells is a prognostic biomarker for progression of chronic kidney disease. The other significant prognostic factor is low estimated glomerular filtration rate at baseline. Although the prediction of progression by TNFRSF6B might be an effect of residual confounding by severity of kidney function, we have confirmed that the associations between high TNFRSF6B expression or low glomerular filtration rate with kidney disease progression are independent of each other (Table 2). Our findings imply that the individual relationship of TNFRSF6B expression in kidneys with progression of chronic kidney disease signifies unique pathophysiologic pathways, and is a marker of the different biological process from glomerular filtration rate. Glomerular filtration rate is currently the gold standard of the functional biomarkers in chronic kidney disease, but it only reflects impaired glomerular and tubular function and could not provide information regarding the underlying pathologic process in kidney disease progression. ${ }^{25}$ Therefore, a newer biomarker for risk stratification to direct management of chronic kidney disease is warranted. Although combined urine protein, estimated glomerular filtration rate and other conventional risk factors has demonstrated acceptable discrimination ( $c$ statistic of 0.76), addition of TNFRSF6B further significantly improve the prediction ability for renal progression ( $c$ statistic of 0.82). Till now, no single marker can identify all patients at increased risks for progressive chronic kidney disease. Combination of several independent risk factors are proposed to better classify patients at higher probability for progression. ${ }^{6}$ Our results showed that high TNFRSF6B expression in renal tubular epithelial cells could serve as a novel tissue marker and further improve predictability on the top of other established risk factors to predict progression of chronic kidney disease.

Cumulative evidence indicates that tubulointerstitial fibrosis is an ultimate common pathway of kidney diseases irrespective of the initial causes. ${ }^{15,16}$ Failure of clearance of the fibroblasts after resolution of the initial insults will lead to the persistence of renal fibrogenesis and the resultant tubulointerstitial fibrosis. ${ }^{17}$ Human renal interstitial fibroblasts constitutively express Fas and are 
Table 2 Association of baseline variables with progression of kidney disease after a median follow-up of 30.5 months using multivariate Cox proportional hazards analysis ${ }^{\mathrm{a}}$

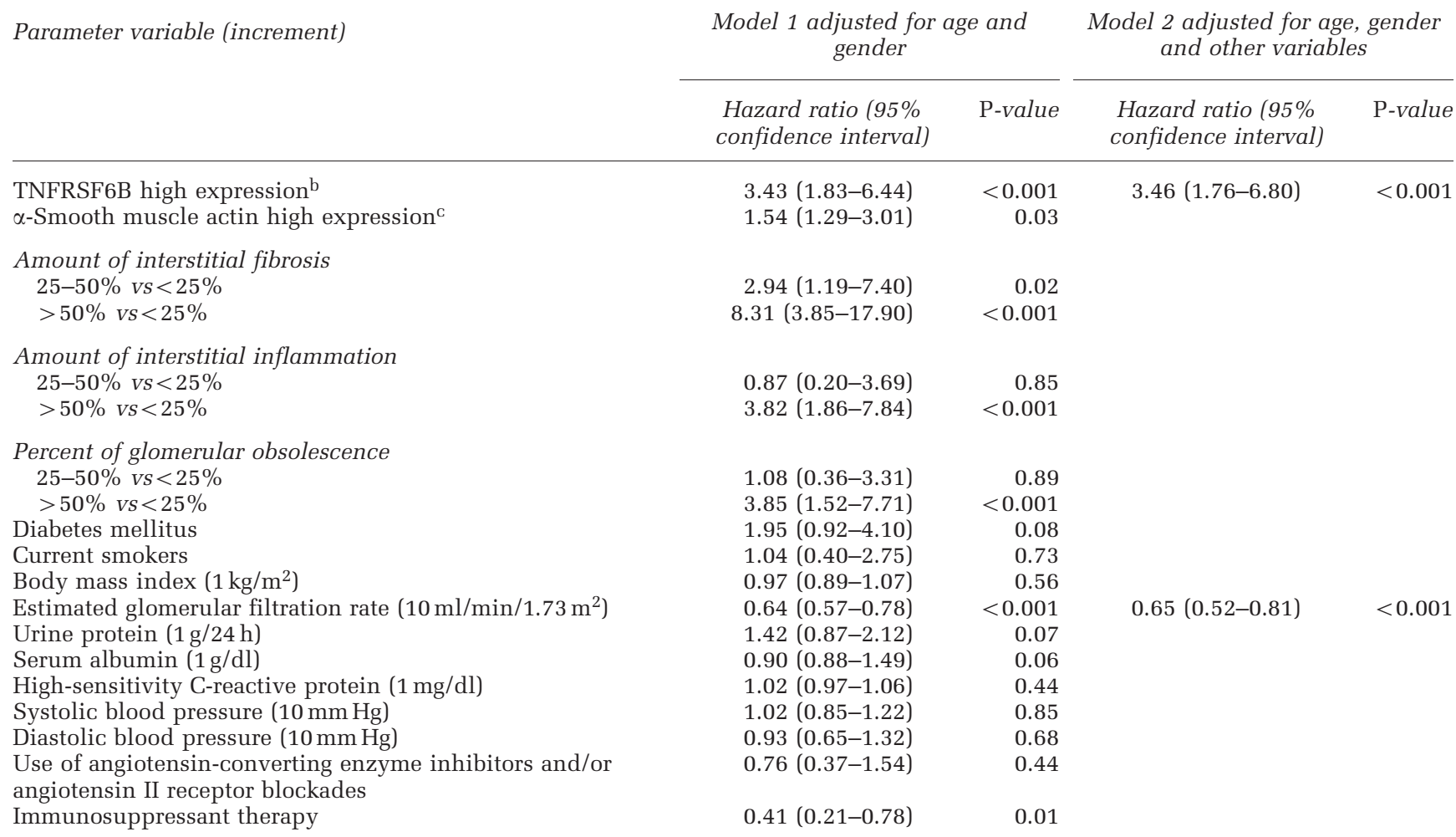

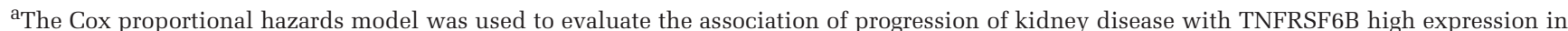
renal tubular epithelial cells and included terms for age, gender, presence of diabetes mellitus, current smoking status, expression of $\alpha$-smooth muscle actin in renal tubular epithelial cells, intensity of interstitial fibrosis and inflammation, percentage of global glomerular obsolescence, estimated glomerular filtration rate, urine protein excretion rate, serum albumin level, immunosuppressant therapy, and use of angiotensinconverting enzyme inhibitors and/or angiotensin-II receptor blockades.

DTNFRSF6B staining: high expression (quantitative immunohistochemical staining value $>0.1119$ ) vs low expression (quantitative immunohistochemical staining value $\leq 0.1119)$.

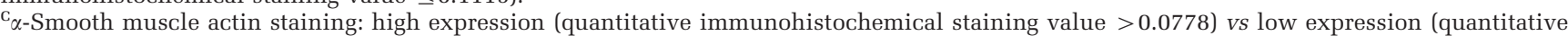
immunohistochemical staining value $\leq 0.0778$ ).

Table 3 Discriminative ability of conventional risk factors and addition of newer biomarkers for prediction of chronic kidney disease progression $^{\mathrm{a}}$

Risk factors and biomarkers

C statistic

Change of c statistic

P-value

(95\% confidence interval)

(95\% confidence interval)

Conventional risk factors only

+ Urine protein

+ Urine protein + estimated glomerular filtration rate

+ Urine protein + estimated glomerular filtration rate +

TNFRSF6B quantitative immunohistochemical staining value
$0.63(0.54$ to 0.73$)$

$0.64(0.54$ to 0.73$)$

$0.76(0.66$ to 0.83$)$

$0.82(0.73$ to 0.88$)$

$\begin{array}{cc}-\overline{0} & \text { Referent } \\ 0.00(-0.04 \text { to } 0.04) & 0.979 \\ 0.05(0.02 \text { to } 0.22) & 0.022 \\ 0.18(0.07 \text { to } 0.29) & 0.001\end{array}$

aRisk prediction was assessed by the $c$ statistic. Each biomarker was stepwise added to model of conventional risk factors to assess the $c$ statistic for predicting progression of chronic kidney disease at 48 months. Conventional risk factors included age, sex, smoking status, diabetes, total cholesterol, and systolic blood pressure.

susceptible to Fas-induced apoptosis. ${ }^{19}$ Hayashi et $a l^{18}$ found that TNFRSF6B offers a survival benefit to synovial fibroblasts in terms of protection against cytotoxic and regulatory effects of Fas. In accordance with these findings, we also showed that TNFRSF6B could protect renal myofibroblasts against Fas-induced apoptosis
(Supplementary Figure S1). Taken collectively, high expression of TNFRSF6B in renal tubular epithelial cells associates with the increased amount of $\alpha$ smooth muscle actin-positive myofibroblasts and interstitial fibrosis probably through the survival advantage to neighboring interstitial myofibroblasts by blocking Fas-induced apoptosis. 
Some limitations should be noted in this study. First, there were no data regarding TNFRSF6B expression in the follow-up renal biopsy specimens. In clinical practice, repeated biopsy was rarely performed except for patients with renal transplantation. We provided the opportunity to figure out the prognosis from a single renal biopsy, which might better fit clinical practice. Second, sample size was relatively small in each subgroup of kidney disease entity. However, using the type of original kidney disease as a covariate in multivariate Cox regression analysis, we still showed the significant power of TNFRSF6B expression to predict progression of chronic kidney disease. Finally, serum TNFRSF6B level was not available in our study. TNFRSF6B in serum is nonspecific for its origins from kidneys or other organs, like lung, colon, spleen, and lymph nodes. ${ }^{8,14}$ In contrast, kidney biopsy remains crucial to accurately assess the expression site of TNFRSF6B, the extent of expression, and histopathology pattern in patients with chronic kidney disease. Therefore, TNFRSF6B expression in kidneys is superior to its serum level in link to the renal outcome.

In summary, TNFRSF6B expression in renal tubular epithelial cells is a novel tissue biomarker for predicting progression of chronic kidney disease. High TNFRSF6B expression in renal tubular epithelial cells is associated with increased myofibroblasts in renal interstitium, and might be a potential therapeutic target for renal fibrosis. Further studies are needed to elucidate the pathophysiologic role of TNFRSF6B in renal fibrogenesis.

\section{Acknowledgements}

This study was supported by the grants from the National Science Council (NSC 96-2628B010 - 001 - MY3), Taipei Veterans General Hospital (V96S5-004, V97S5-004 and V98S5-002), Bureau of Health Promotion, Department of Health (DOH98HP-1110), and Ministry of Education, aim for the Top University Plan. We are grateful to the Division of Experimental Surgery, Department of Surgery for technical assistance in the preparation of kidney biopsy specimens. We are also deeply indebted to PC Lee for her expert secretarial assistance and graphic design.

\section{Disclosure/conflict of interest}

The authors declare no conflict of interest.

\section{References}

1 James MT, Hemmelgarn BR, Tonelli M. Early recognition and prevention of chronic kidney disease. Lancet 2010;375:1296-1309.

2 Go AS, Chertow GM, Fan D, et al. Chronic kidney disease and the risks of death, cardiovascular events, and hospitalization. N Engl J Med 2004;351: 1296-1305.

3 de Zeeuw D, Hillege HL, de Jong PE. The kidney, a cardiovascular risk marker, and a new target for therapy. Kidney Int Suppl 2005;98:S25-S29.

4 Wen CP, Cheng TY, Tsai MK, et al. All-cause mortality attributable to chronic kidney disease: a prospective cohort study based on 462293 adults in Taiwan. Lancet 2008;371:2173-2182.

5 Kent DM, Jafar TH, Hayward RA, et al. Progression risk, urinary protein excretion, and treatment effects of angiotensin-converting enzyme inhibitors in nondiabetic kidney disease. J Am Soc Nephrol 2007;18: 1959-1965.

6 Taal MW, Brenner BM. Renal risk scores: progress and prospects. Kidney Int 2008;73:1216-1219.

7 Levey AS, Cattran D, Friedman A, et al. Proteinuria as a surrogate outcome in CKD: report of a scientific workshop sponsored by the National Kidney Foundation and the US Food and Drug Administration. Am J Kidney Dis 2009;54:205-226.

8 Pitti RM, Marsters SA, Lawrence DA, et al. Genomic amplification of a decoy receptor for Fas ligand in lung and colon cancer. Nature 1998;396:699-703.

9 Yu KY, Kwon B, Ni J, et al. A newly identified member of tumor necrosis factor receptor superfamily (TR6) suppresses LIGHT-mediated apoptosis. J Biol Chem 1999;274:13733-13736.

10 Migone TS, Zhang J, Luo X, et al. TL1A is a TNF-like ligand for DR3 and TR6/DcR3 and functions as a T cell costimulator. Immunity 2002;16:479-492.

11 Lin WW, Hsieh SL. Decoy receptor 3: a pleiotropic immunomodulator and biomarker for inflammatory diseases, autoimmune diseases and cancer. Biochem Pharmacol 2011;81:838-847.

12 Chen J, Zhang L, Kim S. Quantification and detection of DcR3, a decoy receptor in TNFR family. J Immunol Methods 2004;285:63-70.

13 Hung SC, Hsu TW, Lin YP, et al. Decoy receptor 3, a novel inflammatory marker, and mortality in hemodialysis patients. Clin J Am Soc Nephrol 2012;7: 1257-1265.

14 Bai C, Connolly B, Metzker ML, et al. Overexpression of M68/DcR3 in human gastrointestinal tract tumors independent of gene amplification and its location in a four-gene cluster. Proc Natl Acad Sci USA 2000; 97:1230-1235.

15 Risdon RA, Sloper JC, De Wardener HE. Relationship between renal function and histological changes found in renal-biopsy specimens from patients with persistent glomerular nephritis. Lancet 1968;2: 363-366.

16 Zeisberg M, Neilson EG. Mechanisms of tubulointerstitial fibrosis. J Am Soc Nephrol 2010;21:1819-1834.

17 Strutz F, Zeisberg M. Renal fibroblasts and myofibroblasts in chronic kidney disease. J Am Soc Nephrol 2006;17:2992-2998.

18 Hayashi S, Miura Y, Nishiyama T, et al. Decoy receptor 3 expressed in rheumatoid synovial fibroblasts protects the cells against Fas-induced apoptosis. Arthritis Rheum 2007;56:1067-1075.

19 Cheng L, Chen Y. Anti-Fas antibody induces apoptosis in cultured human renal interstitial fibroblasts. Chin Med J (Engl) 2001;114:162-166.

20 Lin SL, Chen RH, Chen YM, et al. Pentoxifylline attenuates tubulointerstitial fibrosis by blocking Smad3/ 4-activated transcription and profibrogenic effects of 
connective tissue growth factor. J Am Soc Nephrol 2005;16:2702-2713.

21 Lee PY, Chien Y, Chiou GY, et al. Induced pluripotent stem cells without c-Myc attenuate acute kidney injury via down-regulating the signaling of oxidative stress and inflammation in ischemia- reperfusion rats. Cell Transplant 2012;21:2569-2585.

22 Hsu YH, Lin WL, Hou YT, et al. Podocalyxin EBP50 ezrin molecular complex enhances the metastatic potential of renal cell carcinoma through recruiting Rac1 guanine nucleotide exchange factor ARHGEF7. Am J Pathol 2010;176:3050-3061.
23 Levey AS, Coresh J, Greene T, et al. Using standardized serum creatinine values in the modification of diet in renal disease study equation for estimating glomerular filtration rate. Ann Intern Med 2006;145: 247-254.

24 Pencina MJ, D’Agostino RB. Overall C as a measure of discrimination in survival analysis: model specific population value and confidence interval estimation. Stat Med 2004;23:2109-2123.

25 Rule AD, Elsherbiny H. Chronic kidney disease: endogenous filtration markers-is it time to move beyond eGFR? Nat Rev Nephrol 2012;8:10-12.

Supplementary Information accompanies the paper on Modern Pathology website (http://www.nature.com/ modpathol) 